\title{
Effect Found in the Combination of Kitasamycin with Other Antibiotics Used against Actinobacillus pleuropneumoniae
}

\author{
Elsa Patricia Flores-Castellanos ${ }^{1}$, Sofía González Gallardo', Jose Ivan Sanchez-Betancourt ${ }^{2}$, \\ Maria Elena Trujillo Ortega ${ }^{2}$, David Quintanar Guerrero'1, Abel Ciprian Carrasco ${ }^{1}$, \\ Susana E. Mendoza Elvira ${ }^{*}$ \\ ${ }^{1}$ Facultad de Estudios Superiores Cuautitlán (FESC), Universidad Nacional Autónoma de México, México City, México \\ ${ }^{2}$ Facultad de Medicina Veterinaria y Zootecnia (FMVZ), Universidad Nacional Autónoma de México, México City, México \\ Email: ${ }^{\star}$ seme@unam.mx
}

How to cite this paper: Flores-Castellanos, E.P., Gallardo, S.G., Sanchez-Betancourt, J.I., Ortega, M.E.T., Guerrero, D.Q., Carrasco, A.C. and Elvira, S.E.M. (2020) Effect Found in the Combination of Kitasamycin with Other Antibiotics Used against Actinobacillus pleuropneumoniae. Journal of Biosciences and Medicines, 8, 63-75. https://doi.org/10.4236/jbm.2020.87007

Received: June 22, 2020

Accepted: July 19, 2020

Published: July 22, 2020

Copyright $\odot 2020$ by author(s) and Scientific Research Publishing Inc. This work is licensed under the Creative Commons Attribution International License (CC BY 4.0).

http://creativecommons.org/licenses/by/4.0/

\begin{abstract}
Background: Combinations of antibiotics are used to increase the therapeutic options via the simultaneous activities of two compounds. The goals of combination therapy are to resolve the bacterial infection while reducing the treatment cost. The use of kitasamycin is used frequently and in addition to other antibiotics, the desired combinations were not found. Methods: Fields strains: we use serotypes 1, 3, 5 y 7. Microbial culturing: The serotypes 1, 3, 5 y 7 was identified. Antibiotics and Reagents: The antibiotics used were kitasamycin; enrofloxacin and oxolinic acid. The following combinations were tested: a) kitasamycin with enrofloxacin; b) kitasamycin with norfloxacin; and c) kitasamycin with oxolinic acid. Preparation of bacterial suspensions: The cultures were adjusted to a concentration of 5 colony-forming units per $\mathrm{ml}$ $(\mathrm{CFU} / \mathrm{ml})$ using the same sterile culture medium as the diluent. Determination of the fractional inhibitory concentrations (FICs): To test each combination and their interactions and to calculate the fractional inhibitory concentration (FIC) of each antibiotic. Calculation of the index of the fractional minimum inhibitory concentration (FMIC): The FMIC index was calculated by applying the following formula: FIC index = FIC of antibiotic A/FIC of antibiotic B. Results: What was found showed that: A combination of kitasamycin and norfloxacin has been shown to have an indifferent effect. A combination of kitasamycin and enrofloxacin had antagonistic effects against all of the Actinobacillus pleuropneumoniae serotypes studied. Finally, a combination of kitasamycin and oxolinic acid had antagonist effects against serotypes 1, 3 and 5 and a synergistic effect against serotype 7 . Conclusion: The "in vitro" combination Kitasamycin-Norfloxacin showed an indifferent effect,
\end{abstract}


and combination Kitasamycin and Enrofloxacin demonstrated an antagonistic interaction between these two antibiotics. A combination of Kitasamycin and Oxolinic Acid had synergistic effect against serotype 7.

\section{Keywords}

Antibiotics, Combination, A. pleuropneumoniae

\section{Introduction}

Actinobacillus pleuropneumoniae is the causative agent of porcine pleuropneumonia (PP), a severe and frequently fatal disease that affects pigs of any age [1]. A variety of antibiotics have been used to control PP, including penicillins, tetracyclines, sulfonamides, erythromycin and new antibiotics such as tiamulin, spectomycin [2] [3] [4]. In recent years, kitasamycin has produced very good results (Asahi Vet. S.A. \& Asahi Chemical Industry Co. LTD., 1995), as have first-generation quinolones, such as nalidixic and oxolinic acid, and fluoroquinolones, such as ciprofloxacin and enrofloxacin [5]. In the antimicrobial therapy of clinical cases of PP on farms, the use of a series of antibiotics and their possible combinations have been reported to find the desired effect. Three types of interactions can result from the combination of two antibiotics: synergism, antagonism and indifference. Parenteral antibiotic treatment has proven to be the most effective strategy to prevent outbreaks of the disease; however, the cost of these treatments is high. The addition of antibiotics to food is of little value during a pleuropneumonia outbreak, as the animals become anorexic, making it difficult for them to consume a therapeutic dose that delivers the minimum inhibitory concentration (MIC) required to eliminate the microorganism; therefore, treatment by this route does not prevent infection, and it can only reduce the severity of the clinical disease [6]. Antibiotic resistant $A$. pleuropneumoniae strains have been isolated, especially in serotypes $1,3,5$ and 7 [7] [8]. Combinations of antibiotics can be used to increase therapeutic options, and a common goal is to identify formulas that can resolve bacterial infections while reducing treatment costs [1] [9]. When two antimicrobial agents are combined, three types of interactions can be observed: synergistic, indifferent and antagonistic. Synergism and antagonism are difficult to predict because the effects vary depending on the microorganism and can occur only within a narrow range of drug concentrations. However, knowledge of the different modes of action of antimicrobial agents allows for the prediction of the effects of various treatments [10] [11].

The results of antibiotic tests can be plotted in graphs known as isobolograms to facilitate the data interpretation [12]. In this study, the in vitro interactions between kitasamycin and three different quinolones-enrofloxacin, floxacin, and oxolinic acid were evaluated against $A$. pleuropneumoniae strains belonging to serotypes 1, 3, 5 and 7 . 


\section{Materials and Methods}

\subsection{Fields Strains}

Actinobacillus strains were isolated from typical cases of contagious pleuropneumonia and were identified as $A$. pleuropneumoniae and identified with specific antisera (according to Mital et al. 1987; Mittal et al., 1992) [13] [14] serotypes $1,3,5$ and 7 . With these serotypes, it was determined the fractional inhibitory concentrations (FICs).

\subsection{Microbial Culturing}

Isolation was performed on brain heart infusion (BHI) agar (Difco Labs., Detroit, Michigan, USA) enriched with 5\% defibrinated calf blood. The colonies obtained on this medium were transferred to BHI media without blood and supplemented with $10 \mathrm{mg} / \mathrm{ml}$ nicotinamide adenine dinucleotide (NAD, Sigma Chemical, St. Louis, Missouri, USA). The cultures were incubated at $37^{\circ} \mathrm{C}$ for 18 h. The identification of suspected A. pleuropneumoniae colonies was based on urease tests, positive CAMP reaction, and positive hemolysis and satellitism. A second set of tests was performed to assess the fermentation of several sugars, including D-xylose, dextrose, fructose, lactose and D-mannitol [14].

\subsection{Antibiotics and Reagents}

The antibiotics used were kitasamycin (Asahi Vet. S.A. and Asahi Chemical Industry Co., LTD.: potency $950 \mu \mathrm{g} / \mathrm{mg}$ ); norfloxacin (Sigma-Aldrich, Life Science, N9890-1G: potency $983.6 \mu \mathrm{g} / \mathrm{mg}$ ); enrofloxacin (Sigma-Aldrich, Life Science, Baytril 17849-5GF: potency $998 \mu \mathrm{g} / \mathrm{mg}$ ); and oxolinic acid (Sigma-Aldrich, Life Science, 00877-5G: potency $890 \mu \mathrm{g} / \mathrm{mg}$ ). The following combinations were tested: 1) kitasamycin with enrofloxacin; 2) kitasamycin with norfloxacin; and 3) kitasamycin with oxolinic acid. Stock solutions $(512 \mu \mathrm{g} / \mathrm{ml})$ of each antibiotic were prepared using sterile distilled water as the diluent, and twofold serial dilutions were performed to obtain solutions of the different combinations over a concentration range of $512 \mu \mathrm{g} / \mathrm{ml}$ to $0.5 \mu \mathrm{g} / \mathrm{ml}$.

\subsection{Preparation of Bacterial Suspensions}

A seed culture of each $A$. pleuropneumoniae serotype was used to inoculate 1 to $25 \mathrm{ml}$ of sterile BHI broth supplemented with Factor V and $10 \mathrm{mg} / \mathrm{ml}$ of nicotinamide adenine dinucleotide (NAD) (Sigma Chemical, St. Louis, Missouri, USA), and the cultures were then incubated at $37^{\circ} \mathrm{C}$ for $20 \mathrm{~h}$ [1]. The cultures were adjusted to a concentration of 5 colony-forming units per $\mathrm{ml}(\mathrm{CFU} / \mathrm{ml})$ using the same sterile culture medium as the diluent.

\subsection{Determination of the Fractional Inhibitory Concentrations (FICs)}

To test each combination and their interactions and to calculate the fractional inhibitory concentration (FIC) of each antibiotic, constant volumes of antibiotic 
(A) were placed in the wells of a sterile 96-well plate (Nunclon, Amsterdam) starting with the lowest concentration at the top and continuing to the highest concentration at the bottom. Subsequently, constant volumes of antibiotic B were placed from left to right, beginning with the lowest concentration. Finally, constant volumes of the bacterial suspension were placed in each well of the plate. Well H1 was left as the culture control, and well H12 was used as the medium control. The plates were then incubated at $37^{\circ} \mathrm{C}$ for $20 \mathrm{~h}$ with gentle shaking. The interpretation of the results was performed as follows: 1) Each well was examined with a light source, and their turbidities were compared with that of well A1, which had a high turbidity due to bacterial growth. All of the wells that showed turbidity were recorded. 2) The minimum inhibitory concentration (MIC) of antibiotic A, which corresponds to the lowest antibiotic concentration that completely inhibits bacterial growth, was determined. 3) The MIC of antibiotic B was determined in the same way. 4) The fractional minimum inhibitory concentration (FMIC) of each of the tested antibiotics was calculated using the formula FMIC = MIC of the antibiotic in combination/MIC of the antibiotic alone [6] [8]

\subsection{Calculation of the Index of the Fractional Minimum Inhibitory Concentration (FMIC)}

The FMIC index was calculated by applying the following formula: FIC index = FIC of antibiotic A/FIC of antibiotic B. FIC indexes less than or equal to 0.5 indicate synergism, FIC indexes between 0.5 and 4 indicate indifference between the antibiotics, and FIC indexes greater than 4 indicate antagonism between the antibiotics [6] [8] [12].

\subsection{Trial Registration}

The manuscript does not contain clinical studies or patient data; the samples used in this study were voluntarily provided by veterinarians and pig farmers.

\section{Results}

\subsection{Combination of Kitasamycin with Enrofloxacin}

The MICs for enrofloxacin were $0.15 \mu \mathrm{g} / \mathrm{ml}$ for serotype $1,0.62 \mu \mathrm{g} / \mathrm{ml}$ for serotype 3 , and $0.31 \mu \mathrm{g} / \mathrm{ml}$ for serotypes 5 and 7 . These results demonstrate the potent antimicrobial action of this fluoroquinolone. The MICs for kitasamycin were $16.0 \mu \mathrm{g} / \mathrm{mg}$ for serotypes 1,3 and 7 and $32.0 \mu \mathrm{g} / \mathrm{ml}$ for serotype 5 . The results demonstrated an antagonistic interaction between these two antibiotics when in combination. The following FICs were determined: for serotype 1, between 2.0 and 96.0; for serotype 3, between 32.25 and 72.0; for serotype 5, between 1.5 and 48.0; and for serotype 7, between 1.5 and 80.0. The analysis of the interactions is summarized in Tables $1(\mathrm{a})$-(d).

\subsection{Combination of Kitasamycin with Norfloxacin}

The MICs for norfloxacin were $0.64 \mu \mathrm{g} / \mathrm{ml}$ for serotypes 1 and 3, $1.28 \mu \mathrm{g} / \mathrm{ml}$ for 
Table 1. Effects of the combination of kitasamycin and enrofloxacin on the growth of $A$. pleuropneumoniae serotypes 1, 3, 5 and 7 (from top to bottom). The tables show all of the calculations performed to determine the FICs of the antibiotics, the FIC index and their respective interpretation in each serotype. (a) A. pleuropneumoniae SEROTYPE 1; (b) $A$. pleuropneumoniae SEROTYPE 3; (c) A. pleuropneumoniae SEROTYPE 5; (d) A. pleuropneumoniae SEROTYPE 7.

(a)

\begin{tabular}{ccccccc}
\hline \multirow{2}{*}{$\begin{array}{c}\text { LIGHT } \\
\text { WELL }\end{array}$} & \multicolumn{2}{c}{ KITASAMICIN } & \multicolumn{2}{c}{ ENROFLOXACIN } & & \\
\cline { 2 - 5 } & $\mu \mathrm{g} / \mathrm{ml}$ & FIC A & $\mu \mathrm{g} / \mathrm{ml}$ & FIC B & & \\
\hline A7 & 16.0 & MIC of A & 0 & MIC of A & MIC of A & MIC of A \\
B7 & 16.0 & 1.0 & 0.15 & 1.0 & 2.0 & $\mathrm{I}$ \\
C13 & 1024 & 64.0 & 0.31 & 2.0 & 66.0 & $\mathrm{~A}$ \\
D13 & 1024 & 64.0 & 0.62 & 4.0 & 68.0 & $\mathrm{~A}$ \\
E13 & 1024 & 64.0 & 1.25 & 8.0 & 72.0 & $\mathrm{~A}$ \\
F13 & 1024 & 64.0 & 2.5 & 16.0 & 80.0 & $\mathrm{~A}$ \\
G13 & 1024 & 64.0 & 5.0 & 32.0 & 96.0 & $\mathrm{~A}$ \\
B1 & 0 & MIC of B & 0.15 & MIC of B & MIC of B & MIC of B \\
\hline
\end{tabular}

(b)

\begin{tabular}{ccccccc}
\hline \multirow{2}{*}{$\begin{array}{c}\text { LIGHT } \\
\text { WELL }\end{array}$} & \multicolumn{2}{c}{ KITASAMICIN } & \multicolumn{2}{c}{ ENROFLOXACIN } & & \\
\cline { 2 - 5 } & $\mu \mathrm{g} / \mathrm{ml}$ & FIC A & $\mu \mathrm{g} / \mathrm{ml}$ & FIC B & & \\
\hline A7 & 16.0 & MIC of A & 0 & MIC of A & MIC of A & MIC of A \\
B10 & 512 & 32.0 & 0.15 & 0.25 & 32.25 & A \\
C10 & 512 & 32.0 & 0.31 & 0.5 & 32.5 & A \\
D12 & 512 & 32.0 & 0.62 & 1.0 & 33.0 & A \\
E12 & 512 & 32.0 & 1.25 & 2.0 & 34.0 & A \\
F13 & 1024 & 64.0 & 2.5 & 4.0 & 68.0 & A \\
G13 & 1024 & 64.0 & 5.0 & 8.0 & 72.0 & A \\
D1 & 0 & MIC of B & 0.62 & MIC of B & MIC of B & MIC of B \\
\hline
\end{tabular}

(c)

\begin{tabular}{ccccccc}
\hline \multirow{2}{*}{$\begin{array}{c}\text { LIGHT } \\
\text { WELL }\end{array}$} & \multicolumn{2}{c}{ KITASAMICIN } & \multicolumn{2}{c}{ ENROFLOXACIN } & \multicolumn{2}{c}{ FIC INDEX INTERPRETATION } \\
\cline { 2 - 5 } & $\mu \mathrm{g} / \mathrm{ml}$ & FIC A & $\mu \mathrm{g} / \mathrm{ml}$ & FIC B & & MIC of A \\
A7 & 32.0 & MIC of A & 0 & MIC of A & MIC of A & I \\
B8 & 32.0 & 1.0 & 0.15 & 0.5 & 1.5 & I \\
C8 & 32.0 & 1.0 & 0.31 & 1.0 & 2.0 & A \\
D9 & 64.0 & 2.0 & 0.62 & 2.0 & 4.0 & A \\
E9 & 64.0 & 2.0 & 1.25 & 4.0 & 6.0 & A \\
F13 & 1024 & 32.0 & 2.5 & 8.0 & 40.0 & A \\
G13 & 1024 & 32.0 & 5.0 & 16.0 & 48.0 & MIC of B \\
C1 & 0 & MIC of B & 0.31 & MIC of B & MIC of B & \\
\hline
\end{tabular}


(d)

\begin{tabular}{ccccccc}
\hline \multirow{2}{*}{$\begin{array}{c}\text { LIGHT } \\
\text { WELL }\end{array}$} & \multicolumn{2}{c}{ KITASAMICIN } & \multicolumn{2}{c}{ ENROFLOXACIN } & \multicolumn{2}{c}{ FIC INDEX INTERPRETATION } \\
\cline { 2 - 5 } A7 & $\mu \mathrm{g} / \mathrm{ml}$ & FIC A & $\mu \mathrm{g} / \mathrm{ml}$ & FIC B & & MIC of A \\
B7 & 16.0 & MIC of A & 0 & MIC of A & MIC of A & I \\
C10 & 16.0 & 1.0 & 0.15 & 0.5 & 1.5 & A \\
D13 & 128 & 8.0 & 0.31 & 1.0 & 9.0 & A \\
E13 & 1024 & 64.0 & 0.62 & 2.0 & 66.0 & A \\
F13 & 1024 & 64.0 & 1.25 & 4.0 & 68.0 & A \\
G13 & 1024 & 64.0 & 2.0 & 8.0 & 72.0 & A \\
C1 & 0 & MIC of B & 0.31 & MIC of B & MIC of B & MIC of B \\
\hline
\end{tabular}

$\mathrm{I}=$ indifference; $\mathrm{A}=$ antagonism; $\mathrm{S}=$ synergism; $\mathrm{MIC}=$ minimum inhibitory concentration; FIC = fractional minimum inhibitory concentration.

serotype 5 , and $0.32 \mu \mathrm{g} / \mathrm{ml}$ for serotype 7 . The MICs of kitasamycin were similar to the previous values. These results revealed an indifferent interaction between these antibiotics when applied in combination against all four serotypes. The following FICs were determined: for serotype 1, between 0.625 and 1.25; for serotype 3, between 0.625 and 1.25; for serotype 5, between 0.5 and 1.0; and for serotype 7, between 1.25 and 5 . It is important to mention that there was some level of antagonism with serotype 5 , which showed an FIC of 5 with MICs of kitasamycin and floxacin of $64.0 \mu \mathrm{g} / \mathrm{ml}$ and $0.32 \mu \mathrm{g} / \mathrm{ml}$, respectively. The mathematical analysis of the data is shown in Tables 2(a)-(d).

\subsection{Combination of Kitasamycin with Oxolinic Acid}

The MIC for kitasamycin was similar to the previous value for serotypes 1, 3 and 7 of A. pleuropneumoniae (i.e., $16.0 \mu \mathrm{g} / \mathrm{ml}$ ), and the MIC against serotype 5 was $32.0 \mu \mathrm{g} / \mathrm{ml}$. In the case of oxolinic acid, the MIC for serotypes 3, 5 and 7 was 2.0 $\mu \mathrm{g} / \mathrm{ml}$, while the MIC for serotype 1 was $1.28 \mu \mathrm{g} / \mathrm{ml}$. The results showed an indifference effect for this combination against serotype 1, with FIC values ranging from 0.75 to 1.25. An antagonistic effect was found for serotypes 3 and 5, with higher FIC values of 18.0 and 6.0, respectively. For serotype 7, the effect was synergistic-indifferent, with one synergistic point. Thus, the concentrations required for a synergistic effect for the combination of kitasamycin and oxolinic acid were $2.0 \mu \mathrm{g} / \mathrm{ml}$ and $0.5 \mu \mathrm{g} / \mathrm{ml}$, respectively. The FIC value was 0.375 , and these data are summarized in Tables 3(a)-(d).

\section{Trial registration}

The manuscript does not contain clinical studies or patient data; the samples used in this study were voluntarily provided by veterinarians and pig farmers.

\section{Discussion}

Porcine pleuropneumonia (PP) was first isolated by Pattison in 1957, and it has 
since been the subject of multiple studies; however, in the late 1970s, it emerged in the international arena as a disease of economic importance in fattening pigs [1]. The etiological agent of PP is A. pleuropneumoniae, which has a worldwide distribution. Currently, 15 serotypes of this microorganism have been recognized, and the various serotypes vary in their degree of virulence and distribution, as first established by Fenwick [15] [16]. The disease is highly contagious

Table 2. Effects of the combination of kitasamycin and norfloxacin on growth of $A$. pleuropneumoniae serotypes 1, 3, 5 and 7 (from top to bottom). The tables show all of the calculations performed to determine the FICs of the antibiotics, the FIC index and their respective interpretation in each serotype. (a) A. pleuropneumoniae SEROTYPE 1; (b) $A$. pleuropneumoniae SEROTYPE 3; (c) A. pleuropneumoniae SEROTYPE 5; (d) A. pleuropneumoniae SEROTYPE 7.

(a)

\begin{tabular}{ccccccc}
\hline \multirow{2}{*}{$\begin{array}{c}\text { LIGHT } \\
\text { WELL }\end{array}$} & \multicolumn{2}{c}{ KITASAMICIN } & \multicolumn{2}{c}{ NORPHLOXACIN } & \multicolumn{2}{c}{ FIC INDEX INTERPRETATION } \\
\cline { 2 - 5 } & $\mu \mathrm{g} / \mathrm{ml}$ & FIC A & $\mu \mathrm{g} / \mathrm{ml}$ & FIC B & & MIC of A \\
\hline A7 & 16.0 & MIC of A & 0 & MIC of A & MIC of A & I \\
B6 & 8.0 & 0.5 & 0.08 & 0.12 & 0.62 & I \\
C6 & 8.0 & 0.5 & 0.16 & 0.25 & 0.75 & I \\
D6 & 8.0 & 0.5 & 0.32 & 0.5 & 1.0 & I \\
E5 & 4.0 & 0.25 & 0.64 & 1.0 & 1.25 & MIC of B \\
E1 & 0 & MIC of B & $\mathbf{0 . 6 4}$ & MIC of B & MIC of B &
\end{tabular}

(b)

\begin{tabular}{|c|c|c|c|c|c|c|}
\hline \multirow{2}{*}{$\begin{array}{l}\text { LIGHT } \\
W E L L\end{array}$} & \multicolumn{2}{|c|}{ KITASAMICIN } & \multicolumn{2}{|c|}{ NORPHLOXACIN } & \multirow{2}{*}{ FIC INDEX } & \multirow{2}{*}{$\begin{array}{c}\text { INTERPRETATION } \\
\mu \mathrm{g} / \mathrm{ml}\end{array}$} \\
\hline & $\mu \mathrm{g} / \mathrm{ml}$ & FIC A & $\mu \mathrm{g} / \mathrm{ml}$ & FIC B & & \\
\hline A7 & 16.0 & MIC of A & 0 & MIC of A & MIC of A & MIC of A \\
\hline B6 & 8.0 & 0.5 & 0.08 & 0.12 & 0.62 & I \\
\hline $\mathrm{C} 6$ & 8.0 & 0.5 & 0.16 & 0.25 & 0.75 & I \\
\hline D6 & 8.0 & 0.5 & 0.32 & 0.5 & 1.0 & I \\
\hline E3 & 4.0 & 0.25 & 0.64 & 1.0 & 1.25 & I \\
\hline E1 & 0 & MIC of B & 0.64 & MIC of B & MIC of B & MIC of B \\
\hline
\end{tabular}

(c)

\begin{tabular}{ccccccc}
\hline \multirow{2}{*}{$\begin{array}{c}\text { LIGHT } \\
\text { WELL }\end{array}$} & \multicolumn{2}{c}{ KITASAMICIN } & \multicolumn{2}{c}{ NORPHLOXACIN } & FIC INDEX & $\begin{array}{c}\text { INTERPRETATION } \\
\mu \mathrm{g} / \mathrm{ml}\end{array}$ \\
\cline { 2 - 5 } A7 & $\mu \mathrm{g} / \mathrm{ml}$ & FIC A & $\mu \mathrm{g} / \mathrm{ml}$ & FIC B & & MIC of A \\
B7 & 32.0 & MIC of A & 0 & MIC of A & MIC of A & I \\
C7 & 16.0 & 0.5 & 0.08 & 0.06 & 0.56 & I \\
D7 & 16.0 & 0.5 & 0.16 & 0.12 & 0.62 & I \\
E7 & 16.0 & 0.5 & 0.32 & 0.25 & 0.75 & I \\
F1 & 16.0 & 0.5 & 0.64 & 0.5 & 1.0 & MIC of B \\
\hline
\end{tabular}


(d)

\begin{tabular}{ccccccc}
\hline \multirow{2}{*}{$\begin{array}{c}\text { LIGHT } \\
\text { WELL }\end{array}$} & \multicolumn{2}{c}{ KITASAMICIN } & \multicolumn{2}{c}{ NORPHLOXACIN } & FIC INDEX & $\begin{array}{c}\text { INTERPRETATION } \\
\mu \mathrm{g} / \mathrm{ml}\end{array}$ \\
\cline { 2 - 5 } A7 & $\mu \mathrm{g} / \mathrm{ml}$ & FIC A & $\mu \mathrm{g} / \mathrm{ml}$ & FIC B & MIC of A \\
B7 & 16.0 & MIC of A & 0 & MIC of A & MIC of A & I \\
C7 & 16.0 & 1.0 & 0.08 & 0.25 & 1.25 & I \\
D9 & 16.0 & 1.0 & 0.16 & 0.5 & 1.5 & A \\
E7 & 16.0 & 4.0 & 0.32 & 1.0 & 5.0 & I \\
D1 & 0 & 1.0 & 0.64 & 2.0 & 3.0 & MIC of B \\
\hline
\end{tabular}

$\mathrm{I}=$ indifference; $\mathrm{A}=$ antagonism; $\mathrm{S}=$ synergism; $\mathrm{MIC}=$ minimum inhibitory concentration; FIC = fractional minimum inhibitory concentration.

Table 3. Effects of the combination of kitasamycin and oxolinic acid on growth of $A$. pleuropneumoniae serotypes 1, 3, 5 and 7 (from top to bottom). The tables show all of the calculations performed to determine the FICs of the antibiotics, the FIC index and their respective interpretation in each serotype. (a) A. pleuropneumoniae SEROTYPE 1; (b) $A$. pleuropneumoniae SEROTYPE 3; (c) A. pleuropneumoniae SEROTYPE 5; (d) A. pleuropneumoniae SEROTYPE 7.

(a)

\begin{tabular}{ccccccc}
\hline \multirow{2}{*}{$\begin{array}{c}\text { LIGHT } \\
\text { WELL }\end{array}$} & \multicolumn{2}{c}{ KITASAMICIN } & \multicolumn{2}{c}{ OXOLYLNIC ACID } & \multicolumn{1}{c}{ FIC INDEX INTERPRETATION } \\
\cline { 2 - 5 } & $\mu \mathrm{g} / \mathrm{ml}$ & FIC A & $\mu \mathrm{g} / \mathrm{ml}$ & FIC B & & MIC of A \\
A7 & 16.0 & MIC of A & 0 & MIC of A & MIC of A & I \\
B7 & 16.0 & 1.0 & 0.08 & 0.06 & 1.06 & I \\
C7 & 16.0 & 1.0 & 0.16 & 0.12 & 1.12 & I \\
D7 & 16.0 & 1.0 & 0.32 & 0.25 & 1.25 & I \\
E5 & 4.0 & 0.25 & 0.64 & 0.5 & 0.75 & MIC of B \\
F1 & 0 & MIC of B & $\mathbf{1 . 2 8}$ & MIC of B & MIC of B & \\
\hline
\end{tabular}

(b)

\begin{tabular}{|c|c|c|c|c|c|c|}
\hline \multirow{2}{*}{$\begin{array}{c}L I G H T \\
W E L L\end{array}$} & \multicolumn{2}{|c|}{ KITASAMICIN } & \multicolumn{2}{|c|}{ OXOLYLNIC ACID } & \multirow{2}{*}{ FIC INDEX } & \multirow{2}{*}{ INTERPRETATION } \\
\hline & $\mu \mathrm{g} / \mathrm{ml}$ & FIC A & $\mu \mathrm{g} / \mathrm{ml}$ & FIC B & & \\
\hline A7 & 16.0 & MIC of A & 0 & MIC of A & MIC of A & MIC of A \\
\hline B11 & 256 & 16.0 & 0.5 & 0.25 & 16.25 & A \\
\hline $\mathrm{C} 11$ & 256 & 16.0 & 1.0 & 0.5 & 16.5 & A \\
\hline D11 & 256 & 16.0 & 2.0 & 1.0 & 17.0 & A \\
\hline E11 & 256 & 16.0 & 4.0 & 2.0 & 18.0 & A \\
\hline F2 & 0.5 & 0.031 & 8.0 & 4.0 & 4.031 & A \\
\hline D1 & 0 & MIC of B & 2.0 & MIC of B & MIC of B & MIC of B \\
\hline
\end{tabular}


(c)

\begin{tabular}{ccccccc}
\hline \multirow{2}{*}{$\begin{array}{c}\text { LIGHT } \\
\text { WELL }\end{array}$} & \multicolumn{2}{c}{ KITASAMICIN } & \multicolumn{2}{c}{ OXOLYLNIC ACID } & & \\
\cline { 2 - 5 } & $\mu \mathrm{g} / \mathrm{ml}$ & FIC A & $\mu \mathrm{g} / \mathrm{ml}$ & FIC B & & \\
\hline A8 & 32.0 & MIC of A & 0 & MIC of A & MIC of A & MIC of A \\
B10 & 128.0 & 4.0 & 0.5 & 0.25 & 4.25 & A \\
C10 & 128.0 & 4.0 & 1.0 & 0.5 & 4.5 & A \\
D10 & 128.0 & 4.0 & 3.0 & 1.0 & 5.0 & A \\
E10 & 128.0 & 4.0 & 4.0 & 2.0 & 6.0 & A \\
D1 & 0 & MIC of B & 2.0 & MIC of B & MIC of B & MIC of B \\
\hline
\end{tabular}

(d)

\begin{tabular}{|c|c|c|c|c|c|c|}
\hline \multirow{2}{*}{$\begin{array}{c}L I G H T \\
W E L L\end{array}$} & \multicolumn{2}{|c|}{ KITASAMICIN } & \multicolumn{2}{|c|}{ OXOLYLNIC ACID } & \multirow{2}{*}{\multicolumn{2}{|c|}{ FIC INDEX INTERPRETATION }} \\
\hline & $\mu \mathrm{g} / \mathrm{ml}$ & FIC A & $\mu \mathrm{g} / \mathrm{ml}$ & FIC B & & \\
\hline A7 & 16.0 & MIC of A & 0 & MIC of A & MIC of A & MIC of A \\
\hline B4 & 2.0 & 0.12 & 0.5 & 0.25 & 0.37 & S \\
\hline $\mathrm{C} 4$ & 2.0 & 0.12 & 1.0 & 0.5 & 0.62 & I \\
\hline D4 & 2.0 & 0.12 & 2.0 & 1.0 & 1.12 & I \\
\hline $\mathrm{E} 4$ & 2.0 & 0.12 & 4.0 & 2.0 & 2.12 & I \\
\hline D1 & 0 & MIC of B & 2.0 & MIC of B & MIC of B & MIC of B \\
\hline
\end{tabular}

$\mathrm{I}=$ indifference; $\mathrm{A}=$ antagonism; $\mathrm{S}=$ synergism; $\mathrm{MIC}=$ minimum inhibitory concentration; FIC = fractional minimum inhibitory concentration.

and fatal if antibiotic treatment is not initiated after the first case in the herd has occurred; therefore, its control requires immediate parenteral antibiotic therapy [1].

Antibiotic combinations are routinely used to treat seriously ill individuals in human clinical contexts and in veterinary medicine. All of the combinations must be empirically evaluated based on general principles derived from daily practice, either based on well-defined control of bacterial growth or on the predicted activities of the antibiotics to be used. In addition, the indiscriminate use of antibiotics and the lack of knowledge about antibiotic combinations, are facts that have contributed to antibiotic resistance and therapeutic failure [1] [3] [5] [17] [18]. In the past, the participation of a clinical microbiology laboratory was rarely required to confirm the possible effects of a combination of antibiotics; however, this has now changed. Production laboratories in veterinary medicine require the assistance of microbiologists to perform in vitro and in vivo tests to predict the effects of combinations of antimicrobial agents. This cooperation leads to optimization of the antibiotic concentrations used for the treatment of bacterial infections such as Porcine Respiratory Syndrome or related conditions in other species [1] [19]. Antibiotics commonly used for PP treatment have become progressively less effective as the strains continue to acquire increased antibiotic resistance, and this outcome has led to the routine use of antibiotic com- 
binations [18]. The experimental design of this study was based on the testing of combinations of antibiotics using an antimicrobial agent from each group with the intent to classify the combinations into interaction categories [6] [20] established that synergistic and antagonistic interactions are difficult to predict because the results vary depending on the microorganism and the bacterial species and due to the fact that the interactions might only occur within a narrow range of antibiotic concentrations. However, knowledge of the different modes of action of antimicrobial agents does not always allow the prediction of the resulting effects [12]. In this study, pairwise combinations of kitasamycin and three different members of the quinolone family (oxolinic acid, floxacin and enrofloxacin), all of which are commonly used for the treatment of PP produced by $A$. pleuropneumoniae serotypes 1, 3, 5 and 7, were tested using the "checkerboard method", which relies on microdilution to establish possible synergy.

With oxolinic acid, an effect was observed that depended on the A.pleuropneumoniae serogroup: for serotype 1 , the interaction was indifferent; for serotypes 3 and 5 it was antagonistic, and for serotype 7 it was synergistic-indifferent. These differences are due to the different resistance patterns mentioned above [2] [3] [7]. In the case of the combination of kitasamycin with floxacin, the effect for all serotypes was indifference, with the exception of serotype 7 , which presented a clear synergistic effect. This finding is in opposition to the antagonistic effect observed for the combination of kitasamycin with enrofloxacin against this serotype, as previously reported by Lewin and Smith [18]. It is not surprising that inhibitors of protein synthesis, such as kitasamycin, and inhibitors of RNA synthesis, such as rifamycin, have antagonistic effects on the bactericidal activity of quinolones, as the target of their action is DNA gyrase. The company Asahi (Asahi Vet. S.A. and Asahi Chemical Industry Co. LTD., 1995) performed studies on pneumonia in pigs and reported MICs for kitasamycin ranging from 1 $\mu \mathrm{g} / \mathrm{ml}$ to $6.25 \mu \mathrm{g} / \mathrm{ml}$ against pathogenic organisms such as $A$. pleuropneumoniae. These data agree with the results obtained in this study.

The data obtained in this study are comparable to a previously reported MIC value [4] for enrofloxacin of $0.06 \mu \mathrm{g} / \mathrm{ml}$ against strains of $A$. pleuropneumoniae from the United States. This value is 2.6 times higher than the value obtained with serotypes 1 and $7(0.15 \mu \mathrm{g} / \mathrm{ml}), 10.4$-fold higher than that for serotype 3 $(0.64 \mu \mathrm{g} / \mathrm{ml})$, and 5.2 -fold higher than that for serotype $5(0.31 \mu \mathrm{g} / \mathrm{ml})$. These values, despite being comparatively high, represent relatively low antibiotic concentrations and thus are consistent with what has been reported for the widely used broad spectrum fluoroquinolones in clinical contexts [18].

The variations in the effects of the same antibiotic against the different $A$. pleuropneumoniae serotypes can be explained based on the acquisition of antibiotic resistance factors; however, it has not been fully proven whether the presence of $\mathrm{R}$ plasmids causes these effects in A. pleuropneumoniae serotypes, since multiresistant $A$. pleuropneumoniae strains have been found, suggesting that resistance is indeed mediated by plasmids [20] (although the authors of this study 
do admit that the results should be confirmed). Similarly, it has been suggested [21] [22] (that this microorganism obtains its antibiotic resistance genes via external sources. One possibility is via plasmid transfer from the Enterobacteriaceae family to susceptible $A$. pleuropneumoniae strains, which could be associated with the presence of a small cryptic plasmid in the recipient strain. Several plasmids have been identified in A. pleuropneumoniae strains: pVM104, pVM105 and pVM106. The pVM104 and pVM106 plasmids are identical with a molecular weight of 2.3 megadaltons, and these plasmids encode sulfonamide and streptomycin resistance; the plasmid pVM105 has a PM of 3.5 megadaltons and encodes sulfonamide and ampicillin resistance. However, none of these plasmids can promote their own transformation [21]. The combinations of antimicrobials and their probable effects on pathogenic microorganisms of veterinary interest presented here indicate that the routine use of antibiotics in veterinary medicine has led to the development of multiresistance that limits their use in the treatment of porcine respiratory syndrome involving porcine pleuropneumonia.

\section{Conclusion}

The combination of kitasamycin with enrofloxacin demonstrated an antagonistic interaction between these two antibiotics when in combination. The combination of kitasamycin with norfloxacin revealed an indifferent interaction between these antibiotics when applied in combination against all four serotypes. Combination of kitasamycin and oxolinic acid had synergistic effect against serotype 7. The results showed an indifference effect for this combination against serotype 1, an antagonistic effect was found for serotypes 3 and 5 , for serotype 7 , the effect was synergistic-indifferent, with one synergistic point.

\section{Acknowledgements}

The authors wish to thank Eng. Fernando Sotres Carreras, Eng. Draucin Celis for their technical support.

\section{Authors' Contributions}

Experiments were designed by ACC and SEME, and carried out by EPFC. Execution of the study was supervised by ACC and SEME. All the authors participated in the manuscript preparation which was directed by SEME. The final manuscript was approved by all the authors.

\section{Funding}

Grants: FESC-UNAM (PIAPI2032).

\section{Conflicts of Interest}

The authors declare no conflicts of interest regarding the publication of this paper. 


\section{References}

[1] Gottschalk, M. and Taylor, D. (2019) Actinobacillus pleuropneumoniae. In: Diseases of Swine, 11th Edition, Wiley Blackwell Publishing, Hoboken, 749-766.

[2] Vaillancourt, J.P., Higgins, R., Martineau, G.P., Mittal, K.R. and Lariviere, S. (1988) Changes in the Susceptibility of Actinobacillus pleuropneumoniae to Antimicrobial Agents in Quebec (1981-1986). Journal of the American Veterinary Medical Association, 193, 470-473.

[3] Evans, R.A., Unhenhopp, E.K., Hoffman, L.J., Yancey, R.J. and Paulissen, J.B. (1988) Ceftiofur Hydrochloride, a New Broad-Spectrum Cephalosporin: Effectiveness against Induced Haemophilus Pleuropneumoniae of Growing Swine. Proceeding of the 11 th International Pig Veterinary Society, Lausanne, 1-5 July 1990, 94.

[4] Asahi Vet, S.A. and Asahi Chemical Industry Co., Ltd. (1995) Kitasamycin, Macrolide Antibiotic. Animal Health Use. 24-26.

[5] Prescott, J.F. and Yielding, K.M. (1990) In Vitro Susceptibility of Selected Veterinary Bacterial Pathogens to Ciprofloxacin, Enrofloxacin and Norfloxacin. Canadian Journal of Veterinary Research, 54, 195-197.

[6] Pijpers, A., Vernooy, J., van Leengoed, L. and Verheijden, J. (1990) Feed and Water Consumption in Pigs Following an Actinobacillus pleuropneumoniae Challenge. Proceedings of the International Pig Veterinary Society, 11 th Congress, Lausanne, 39.

[7] Berenbaum, M.C. (1978) A Method for Testing for Synergy with Any Number of Agents. The Journal of Infectious Diseases, 137, 122-130. https://doi.org/10.1093/infdis/137.2.122

[8] Gilbride, K.A. and Rosendal, S. (1984) Antimicrobial Susceptibility of 51 Strains of Haemophilus Pleuropneumoniae. Canadian Journal of Comparative Medicine, 48, 47-50.

[9] White, R.L., Burgess, D.S., Mandaru, M. and Bosso, J.A. (1996) Comparison of Three Different in Vitro Methods of Detecting Synergy: Time Kill, Checkerboard and E Test. Antimicrobial Agents and Chemotherapy, 40, 1914-1918. https://doi.org/10.1128/AAC.40.8.1914

[10] Blaser, J. (1991) Interactions of Antimicrobial Combinations in Vitro: The Relativity of Synergism. Scandinavian Journal of Infectious Diseases. Supplementum, 74, 71-79.

[11] Hua, L., Hilliard, J.J., Shi, Y., Tkaczyk, C., Cheng, L., Yu, X., Datta, V., Ren, S., Feng, H., Zinsou, R., Keller, A., O’Day, T., Du, Q., Cheng, L., Damschroder, M., Robbie, G., Suzich, J. and Stover, C.K. (2014) Assessment of an Anti-Alpha-Toxin Monoclonal Antibody for Prevention and Treatment of Staphylococcus aureus-Induced Pneumonia. Antimicrobial Agents and Chemotherapy, 58, 1108-1117. https://doi.org/10.1128/AAC.02190-13

[12] Tallarida, R.J. (2001) Drug Synergism: Its Detection and Applications. Journal of Pharmacology and Experimental Therapeutics, 298, 865-872.

[13] Mittal, K., Higgins, R. and Lariviere, S. (1987) An Evaluation of Agglutination and Coagglutination Techniques for Serotyping of Haemophilus pleuropneumoniae Isolates. American Journal of Veterinary Research, 48, 219-226.

[14] Mittal, K., Higgins, R., Lariviere, S. and Nadeau, M. (1992) Serological Characterization of Actinobacillus pleuropneumoniae Strains Isolated from Pigs in Quebec. Veterinary Microbiology, 32, 135-148.

https://doi.org/10.1016/0378-1135(92)90101-X 
[15] Fenwick, B. (1990) Virulence Attributes of the Liposaccharides of the HAP Group Organisms. Canadian Journal of Veterinary Research, 54, S28-S32.

[16] Blackall, P., Klaasen, H., Van Den Bosch, H., Kuhnert, P. and Frey, J. (2002) Proposal of a New Serovar of Actinobacillus pleuropneumoniae: Serovar 15. Veterinary Microbiology, 84, 47-52. https://doi.org/10.1016/S0378-1135(01)00428-X

[17] Hsu, F. (1990) Evaluation of Linco-Spectin Sterile Solution and Linco-Spectin 44 Premix in the Treatment of Haemophilus Pleuropneumoniae. Proceedings of the International Pig Veterinary Society, 11 th Congress, Lausanne, 15.

[18] Lewin, C. and Smith, J. (1989) Interactions of the 4-Quinolones with Other Antibacterials. Journal of Medical Microbiology, 29, 221-227.

https://doi.org/10.1099/00222615-29-3-221

[19] Holmer, Y., Salomonsen, C., Jorsal, S.E., Astrup,, L.B., Jensen,, V.F., Borck Høg, B. and Pedersen, K. (2019) Antibiotic Resistance in Porcine Pathogenic Bacteria and Relation to Antibiotic Usage I. BMC Veterinary Research, 15, 449. https://doi.org/10.1186/s12917-019-2162-8

[20] Nadeau, M., Lariviere, S., Higgins, R. and Martineau, G. (1988) Minimal Inhibitory Concentrations of Antimicrobial Agents against Actinobacillus pleuropneumoniae. Canadian Journal of Veterinary Research, 52, 315-318.

[21] Hirsh, D., Martin, L. and Libal, M. (1982) Plasmid-Mediated Antimicrobial Resistance in Haemophilus Pleuropneumoniae. American Journal of Veterinary Research, 43, 269-272.

[22] Willson, P.J., Deneer, H., Potter, A. and Albritton, W. (1989) Characterization of a Streptomycin-Sulfonamide Resistance Plasmid from Actinobacillus pleuropneumoniae. Antimicrobial Agents and Chemotherapy, 33, 235-238.

https://doi.org/10.1128/AAC.33.2.235 